\title{
La valutazione del patrimonio museale: il caso del Museo di Storia Naturale di Firenze
}

Giacomo Manetti*, Massimo Valeri* *

\section{Riassunto}

Il presente lavoro mira ad individuare le prassi per la valutazione dei beni culturali di proprietà delle pubbliche amministrazioni attraverso lo studio del caso del Museo di Storia Naturale di Firenze.

Dopo aver esaminato le principali indicazioni della prassi e della dottrina in tema di valutazione dei beni culturali, gli autori affrontano un caso di studio nel quale è stato adottato, per l'inventariazione dell'intero patrimonio museale, uno dei metodi in precedenza discussi, ossia la stima degli esperti, quale proxy del valore di mercato delle collezioni. Con riferimento al caso di studio si indicano le motivazioni a supporto del metodo adottato e le difficoltà incontrate nella conduzione del processo valutativo.

Dall'indagine condotta emergono alcuni limiti ed alcuni potenziali sviluppi della ricerca, evidenziati nelle conclusioni, sui criteri di valutazione del patrimonio museale.

Parole chiave: Patrimonio museale, beni culturali pubblici, valutazione degli esperti, valore di mercato, Museo di Storia Naturale di Firenze.

Articolo ricevuto: dicembre 2011, accettato: luglio 2012

The valuation of the museum heritage: the case of Natural History Museum of Florence

\section{Abstract}

This work aims to determine the practices in the valuation of public heritage assets by analyzing the case of Natural History Museum of Florence.

After examined the main recommendations in the valuation of cultural assets from practice and theory, the authors are checking the research hypothesis that monetary

* Ricercatore presso l’Università degli Studi di Firenze. E-mail: giacomo.manetti@unifi.it.

** Professore Associato presso l’Università degli Studi di Napoli Parthenope. E-mail: massimo.valeri@uniparthenope.it. 
assessment of collections may improve the discharge of internal and external accountability duties of the museum, by screening the methods useful for this goal. Among these methods, a particular attention is dedicated to the expert appraisal as a proxy of the collections market value.

The case study, that has been selected because of its criticality, confirms the research hypothesis, even if some limitations and potential developments emerge from the analysis and are discussed in the conclusions.

Key words: Museum heritage, public heritage assets, expert assessment, market value, Contingent Valuation Method, Natural History Museum of Florence.

First submission: december 2011, accepted: july 2012

\section{Premessa $^{1}$}

La presente ricerca mira ad esaminare le principali proposte metodologiche avanzate dalla prassi contabile e dalla dottrina per la valutazione dei beni culturali di proprietà pubblica, analizzando la scelta compiuta in tale ambito in una realtà significativa quale il Museo di Storia Naturale di Firenze.

$\mathrm{Su}$ un piano generale, la determinazione e l'iscrizione del valore di tali beni nei prospetti contabili di bilancio assumono una particolare rilevanza in relazione all'obiettivo di fornire una rappresentazione veritiera e corretta della situazione patrimoniale di qualsiasi organizzazione, pubblica o privata (profit e non profit), per cui essi costituiscono asset significativi ${ }^{2}$.

Con riguardo al settore pubblico, ambito di riferimento della nostra analisi, l'effettuazione delle operazioni di recognition, valuation e disclosure dei beni culturali si rivela una scelta coerente con la recente evoluzione del ruolo degli strumenti di reporting delle aziende pubbliche, i quali devono essere in grado di fornire un'efficace risposta ai mutati fabbisogni informativi, interni ed esterni. Questa evoluzione, che si inserisce nel più ampio processo di riforma organizzativa e manageriale della pubblica amministrazione nel nostro Paese, dovrebbe condurre - tra l'altro - all'offerta di accurate informazioni economico-patrimoniali relative alla generalità degli asset controllati, per migliorare le

1. Nonostante il presente lavoro sia frutto di una comune attività di ricerca, i paragrafi Premessa, 1 e 2 sono attribuibili a Massimo Valeri, mentre i paragrafi 3, 3.1 e Conclusioni sono attribuibili a Giacomo Manetti.

2. Con riferimento alle organizzazioni museali, si segnala la tendenza in atto ad una più ampia diffusione di strumenti di comunicazione esterna in grado di fornire una rappresentazione efficace e trasparente delle operazioni della gestione sotto il profilo economico, finanziario e patrimoniale. Tale tendenza trova espressione nel Decreto Ministeriale del 10 maggio 2001 "Atto di indirizzo sui criteri tecnico-scientifici e sugli standard di funzionamento e sviluppo dei musei", il quale nell'Ambito II dedicato all'Assetto finanziario raccomanda la progressiva introduzione del bilancio di esercizio in tutti i musei in sostituzione dell'attuale rendicontazione amministrativa. Circa l'applicazione degli standard di funzionamento e sviluppo agli istituti museali, si veda Valeri (2006). 
scelte di allocazione delle risorse da parte degli organi di governo e per far fronte alle accresciute esigenze di accountability nei confronti di molteplici stakeholder (cittadini, fornitori, finanziatori e dipendenti).

Tuttavia, lo svolgimento delle suddette operazioni incontra non pochi ostacoli. Se alcuni di questi sono imputabili a condizioni specifiche del contesto organizzativo (si pensi alle difficoltà di reperire dati economico-patrimoniali in presenza di un sistema di contabilità finanziaria o alla scarsa sensibilità alla problematica delle valutazioni di bilancio di taluni direttori di musei); altri ostacoli sono ascrivibili alle stesse caratteristiche intrinseche di certi beni culturali (specialmente quelli di carattere artistico) per la cui stima può rivelarsi poco significativa l'applicazione dei tradizionali criteri di valutazione delle immobilizzazioni. Si evidenziano, inoltre, limitati contributi scientifici e di carattere normativo, tecnico e giuridico, di approfondimento della materia.

Partendo da tali considerazioni, il primo obiettivo del presente studio è quello di analizzare i principali metodi di valutazione del patrimonio museale, evidenziandone punti di forza e di debolezza. In proposito, si fa riferimento soprattutto ai metodi proposti dagli standard setter nazionali e internazionali nell'ambito di raccomandazioni che hanno ad oggetto il trattamento contabile degli heritage asset, non trascurando, peraltro, possibili soluzioni metodologiche alternative, quali i modelli di analisi econometrica delle preferenze espresse e, in particolare, il metodo della contingent valuation.

Il secondo obiettivo concerne la verifica dell'effettivo utilizzo di uno dei metodi raccomandati dalla prassi - la stima degli esperti quale proxy del valore di mercato - nella valutazione del patrimonio museale nel Museo di Storia Naturale di Firenze. Il case study è stato condotto attraverso due interviste con il direttore del museo e lo studio delle relazioni tecniche di inventariazione delle collezioni redatte dai curatori delle diverse sezioni.

Pertanto, nel prosieguo del lavoro analizzeremo le caratteristiche dei beni culturali e le modalità della loro valutazione ed eventuale rappresentazione in bilancio secondo la prassi contabile e la dottrina nazionale ed internazionale, riportando successivamente i risultati del caso di studio.

Infine, nelle conclusioni esprimeremo un giudizio sui metodi applicabili per la valutazione del patrimonio museale e sull'approccio seguito nel caso di studio, evidenziando i limiti della ricerca ed individuando alcuni suoi possibili sviluppi.

\section{Definizione e caratteristiche rilevanti dei beni culturali}

Secondo il Codice dei beni culturali e del paesaggio (D.Lgs. 22/2004, modificato dal D.Lgs 156/2006, dal D.Lgs. 157/2006 e dal D.Lgs. 63/2008), il patrimonio culturale è costituito da almeno due categorie di beni: i beni culturali (come ad esempio le raccolte dei musei, gli archivi storici, le cose immo- 
bili e mobili di valore culturale) e quelli paesaggistici (quali siti archeologi, paesaggi, territorio costiero, laghi, fiumi). In particolare, i primi sono "immobili e mobili che presentano interesse artistico, storico, archeologico o etnoantropologico, archivistico e bibliografico e le altre cose individuate dalla legge [...] quali testimonianze aventi valore di civiltà" (art. 2, comma 2).

Tra questi distinguiamo beni culturali mobili e immobili (nonché musei, biblioteche ed archivi) appartenenti ${ }^{3}$ :

a) allo Stato, alle regioni e agli altri enti pubblici territoriali, che si caratterizzano per il fatto di essere beni demaniali, inalienabili e istituzionalmente destinati al godimento pubblico (art. 822 cod. civ. e artt. 53 e 54 cod. beni cult.);

b) ad enti ed istituti pubblici differenti da quelli del punto a), i quali fanno parte del patrimonio indisponibile e, come tali, non possono essere sottratti alla loro funzione pubblica (art. $830 \mathrm{cod}$. civ.);

c) a privati, che siano oggetto di tutela e ai quali siano stati estesi i diritti demaniali per assoggettarli ad usi pubblici per il loro interesse artistico e storico;

d) a privati, non soggetti a vincoli di tutela.

Con riferimento ai beni di proprietà pubblica individuati nei precedenti punti a) e b), i quali costituiscono l'oggetto specifico di analisi nel nostro studio, emerge la finalità sociale per cui questi sono detenuti. Essi, compatibilmente con le esigenze di uso istituzionale e di tutela, sono destinati alla fruizione della collettività e, conseguentemente, sono sottoposti ad un'adeguata attività di protezione e conservazione in modo tale da garantire lo svolgimento della loro funzione per un tempo indefinito (Barton, 2005).

Da quanto detto, si evincono alcune caratteristiche fondamentali degli heritage asset, le quali assumono una particolare rilevanza anche al fine del loro trattamento contabile ${ }^{4}$.

Un primo elemento significativo è rappresentato dall' inalienabilità. Questa caratteristica dei beni culturali potrebbe far presupporre che non si tratti di asset aziendali, poiché l'impossibilità della cessione sarebbe equiparabile alla rinuncia al beneficio economico da loro derivante. Di conseguenza, essi non dovrebbero essere rappresentati in bilancio come parte del patrimonio aziendale, poiché l'organizzazione che li possiede non sarebbe in grado di disporne

3. Per approfondimenti sulla disciplina giuridica italiana in tema di beni culturali e di musei cfr. Barbati, Cammelli e Sciullo (2011), Ferretti (2011). Si vedano, inoltre i numerosi contributi di Cammelli pubblicati sulla AEDON, Rivista di Arti e Diritto On Line, il Mulino, Bologna.

4. In merito alle problematiche relative al trattamento contabile dei beni culturali si veda: Barton (2005); Carman, Carnegie e Wolnizer (1999); Carnegie e Wolnizer (1995 e 1999); Christiansen (2004); Hone (1997); Hooper, Kearins e Green (2005); Lacerra (2009); Micallef e Peirson (1997); Onesti e Romano (2004); Porter (2004) e Romolini (2007). 
liberamente. Tuttavia, in proposito si osserva che, in base all'impostazione dello IASB (International Accounting Standard Board, Framework 1989) e dell'IPSASB (International Public Sector Accounting Standard Board) ${ }^{5}$, non è tanto il diritto di proprietà che qualifica un'attività, quanto piuttosto il controllo dei benefici derivanti dal possesso e il diritto d'uso di un bene, a prescindere dal titolo giuridico in virtù del quale il bene è detenuto.

Pertanto, l'inalienabilità di per se stessa non deve essere considerata un ostacolo al riconoscimento degli heritage asset in bilancio nell'ambito dell' attivo patrimoniale. Ad ogni modo, prendendo anche spunto dalla proposta formulata nel Consultation Paper emanato nel 2006 dall'IPSASB (IFAC/IPSA$\mathrm{SB}, 2006$ ) di distinguere i beni culturali pienamente disponibili da quelli su cui gravano restrizioni alla loro disponibilità, appare opportuno che sia specificato - all'interno del patrimonio - se gli asset in questione siano alienabili oppure no.

Peraltro, si osserva che per l'inclusione degli heritage asset tra le attività è necessario che dal loro possesso derivino per l'ente benefici economici futuri.

$\mathrm{Al}$ riguardo, i principali standard setter internazionali (IPSASB, AASB e ASB - Accounting Standards Board $)^{6}$ che si sono occupati della contabilizzazione degli asset in questione, evidenziano che $\mathrm{i}$ beni in discorso rivestono generalmente una particolare utilità in relazione agli obiettivi dell'organizzazione e che tale utilità è esprimibile in termini di flussi finanziari.

In proposito, si rileva che gli heritage asset sono essenziali per lo svolgimento dell'attività dell'ente, qualora la sua finalità istituzionale sia quella della loro conservazione e valorizzazione (ASB, 2009, pp. 15-16). Inoltre, la loro esposizione al pubblico esercita una certa attrattività, richiamando in alcuni casi presso la struttura ospitante un ampio numero di visitatori-paganti. $\mathrm{Ne}$ deriva che l'utilità in discorso è suscettibile di tradursi in termini monetari.

Tuttavia, tenendo in considerazione la finalità sociale che caratterizza l'attività dell'ente pubblico, occorre rilevare che i beni in oggetto sono in qualche caso messi a disposizione del pubblico gratuitamente o più spesso mediante il pagamento di un prezzo simbolico, spesso inferiore al costo di produzione del servizio museale. Il provento da bigliettazione, in altri termini, non può essere rappresentativo dei servizi potenziali offerti dai beni conservati e/o esposti, né della loro utilità per l'organizzazione.

5. L'IPSASB è un organismo internazionale che opera in seno all'IFAC e deputato all'emissione degli standards contabili applicabili al settore pubblico.

6. L'Accounting Standard Board (ASB) è lo standard setter deputato all'emanazione dei principi contabili per le imprese, le aziende non profit di diritto privato e le pubbliche amministrazioni centrali e locali nel Regno Unito. L'ASB, tuttavia, definisce norme contabili per le imprese; esso attribuisce alla Charity Commission la facoltà di emanare gli standard contabili per le aziende non profit e ai Chartered Institute of Public Finance (CIPFAA) e Local Authority Accounts and Advisory Committe (LASAAC) il potere di emanare le norme contabili rispettivamente per le aziende pubbliche britanniche e scozzesi. Cfr. Pozzoli (2005, p. 120). 
In relazione a queste criticità alcuni autori (Christiansen, 2004; Carnegie e Wolnizer, 1999) hanno sollevato questioni in merito all'opportunità e alla possibilità di inserire i beni culturali in bilancio. Di contro, altri studiosi (Micallef e Peirson, 1997; Porter, 2004), sebbene riconoscano l'ipotesi di assenza di flussi di cassa positivi direttamente imputabili agli heritage asset, ritengono l'utilità che questi ultimi rivestono per il conseguimento delle finalità sociali degli enti pubblici un requisito sufficiente ad attribuire agli stessi beni la qualifica di attività patrimoniali.

Di fronte alle inevitabili difficoltà che si incontrano nel trattamento contabile degli heritage asset, la prassi contabile internazionale ha espresso un chiaro orientamento a favore della loro inclusione nello stato patrimoniale, in considerazione dei benefici che ne derivano sotto il profilo dell'informativa esterna e interna dell' organizzazione ${ }^{7}$. Questa scelta consente infatti di fornire dati più completi sulla situazione patrimoniale dell'ente pubblico a favore della generalità degli stakeholder e, nello specifico, dei contribuenti e dei finanziatori, i quali possono così essere in grado di valutare il comportamento del management nella gestione delle risorse a sua disposizione. Inoltre, le informazioni sulla natura, il valore monetario, il livello delle acquisizioni e il grado di utilizzo degli heritage asset da inserire in bilancio possono offrire utili indicazioni per lo svolgimento di attività di decision making e di performance measurement da parte dello stesso management (Hone, 1997; Barton, 2005).

Infine, è opportuno considerare altre caratteristiche rilevanti dei beni culturali che, pur non ponendo in discussione il rispetto dei requisiti necessari per il loro riconoscimento in bilancio come asset patrimoniali, hanno evidenti implicazioni con riguardo alla determinazione del loro valore monetario.

Tali beni si configurano come opere uniche e non replicabili.

Il loro valore di natura culturale, ambientale e storico è improbabile che possa essere pienamente espresso attraverso un prezzo di mercato.

Inoltre, può risultare estremamente difficile, se non impossibile, stimare la loro vita utile. Oltretutto, il loro valore finanziario, se quantificabile, può incrementare (come del resto decrescere per effetto di fluttuazioni del gusto, revisioni di attribuzioni, ecc.) nel corso del tempo, indipendentemente dal loro stato conservativo.

In definitiva, l'effettuazione della valutazione degli heritage asset risulta un'attività estremamente complessa. Tale complessità è accentuata nel caso in cui il singolo ente pubblico possieda un importante patrimonio culturale, all'interno del quale si trovino numerosi beni, acquisiti nel corso di molti anni, attraverso varie modalità (acquisti a titolo oneroso, donazioni, lasciti o seque-

7. Tali asset, inoltre, generano oneri periodici legati, a titolo esemplificativo, alla loro conservazione e esposizione. Pertanto, la loro inclusione nell'attivo dello stato patrimoniale appare opportuna anche perché creerebbe un legame fra tali poste contabili del conto economico ed i beni ai quali gli oneri in questione si riferiscono nell'attivo dello stato patrimoniale. 
stri), in relazione alle quali risultino applicabili differenti criteri o, addirittura, metodologie valutative.

\section{I metodi applicabili per la valutazione dei beni culturali}

Secondo il dettato normativo, la questione della valutazione dei beni culturali si pone esclusivamente per quegli enti pubblici che hanno sostituito o integrato la contabilità finanziaria ${ }^{8}$, nell'ambito della quale tutte le operazioni della gestione (comprese quelle riguardanti gli asset patrimoniali) sono rilevate esclusivamente con riferimento all'aspetto (appunto) finanziario, con un sistema di accrual accounting, che si basa sul principio della competenza economica. Peraltro, anche con l'adozione di quest'ultimo sistema, non è facile superare le innegabili difficoltà che si incontrano nella determinazione di un valore attendibile e significativo per gli heritage asset.

A questo proposito, i principali standard setter nazionali e internazionali, sia pure muovendosi da impostazioni differenti, hanno cercato di affrontare le problematiche relative alle operazioni di recognition, valuation e disclosure dei beni culturali ${ }^{9}$.

In particolare, l'IPSASB richiede nel già citato Consultation Paper che gli heritage asset siano rappresentati in bilancio attraverso valori che forniscano informazioni utili e rilevanti, in considerazione del servizio potenziale e dei benefici in essi incorporati (IFAC/IPSASB, 2006, par. 3.16). A questo proposito, viene stabilito che la valutazione debba avvenire sulla base del valore corrente di mercato, in quanto risulta più significativo rispetto al costo storico. Tale valore deve essere appositamente determinato, laddove possibile ${ }^{10}$, per tutti i beni culturali ricevuti a seguito di donazione e per quelli acquisiti a titolo oneroso negli anni precedenti rispetto all'esercizio a cui si riferisce il bilancio. Invece, per i beni acquistati nell'ultimo periodo amministrativo, il valore corrente può essere rappresentato dal costo sostenuto per l'acquisizione (IFAC/IPSASB, 2006, par. 4.6).

8. La contabilità finanziaria del settore pubblico in Italia non deve essere confusa con la contabilità a base monetaria. Quest'ultima rileva solamente i movimenti di cassa, mentre la contabilità finanziaria registra anche operazione aventi natura squisitamente finanziaria come, ad esempio, crediti e debiti. La contabilità finanziaria italiana può essere più propriamente definita come modified accrual accounting. Pozzoli (2005, pp. 70-71). Sullo stesso argomento si veda anche: Caperchione (2001); Lüder e Jones (2003).

9. Un quadro delle informazioni, dei requisiti per l'iscrizione in bilancio e dei criteri per la misurazione del valore degli heritage asset fissati dai principali standard setter nazionali e internazionali viene fornito in IFAC/IPSASB (2006, pp. 58-63).

10. Peraltro, nel Consultation Paper, l'IPSASB tiene conto delle difficoltà di valutazione dei beni culturali, stabilendo che un ente possa non essere in grado di ottenere le informazioni necessarie per determinare il valore di mercato degli heritage asset. In questo caso, esso non dovrà procedere alla capitalizzazione, purché fornisca nelle note al Bilancio tutte le informazioni sufficienti a spiegare la scelta effettuata (IFAC/IPSASB 2006, parr. 4.12-4.13). 
È opportuno rilevare che, nell'ambito del documento dell'IPSASB, sono illustrati tre differenti approcci per il trattamento contabile dei beni culturali:

- il non-capitalisation approach, il quale stabilisce che, qualora non sia possibile o conveniente (confrontando il beneficio sotto il profilo informativo con il costo, principalmente in termini di tempo, per l'ottenimento di valori attendibili e significativi) assegnare un valore alla maggioranza (per valore) dei beni culturali posseduti, non si debba procedere alla capitalizzazione di nessuno dei beni in questione;

- il mixed capitalisation approach, che prevede la capitalizzazione dei soli beni per cui è possibile e non eccessivamente oneroso effettuare la relativa valutazione;

- il full capitalisation approach, il quale richiede l'iscrizione nell'attivo patrimoniale del valore di tutti gli heritage asset.

Pur tenendo conto delle difficoltà insite nella valutazione dei beni culturali, l'IPSASB accorda la propria preferenza al terzo metodo, proponendo in particolare di abbandonare quello misto (mixed capitalisation approach), previsto dall'FRS 15 (ASB, 1999), in quanto esso produce informazioni incomplete per gli utilizzatori in ragione dell'applicazione di comportamenti diversi nel trattamento contabile delle stesse tipologie di beni.

Il progetto attuato dall'IPSASB su impulso dell' ASB (Accounting Standards Board) è attualmente interrotto. Tuttavia, a partire dai risultati del medesimo processo di consultazione, si è giunti all'emanazione nel giugno del 2009 nel Regno Unito del FRS (Financial Reporting Standard) n. 30 (ASB, 2009), il primo standard interamente dedicato al trattamento contabile degli heritage asset.

Il FRS 30 stabilisce una serie di requisiti informativi riguardanti il valore e il contenuto delle collezioni da inserire nei conti annuali relativi ai periodi amministrativi che si aprono a partire dal $1^{\circ}$ aprile 2010 .

Con il fine di incoraggiare l'ampliamento del livello di disclosure da parte delle organizzazioni che possiedono beni culturali, l'ASB ha cercato di tenere conto delle difficoltà pratiche insite nella loro valutazione, da un lato, abbandonando alcune impostazioni più radicali emerse nel corso del processo di consultazione condotto dall'IPSASB e, dall'altro, dimostrando una certa elasticità nella determinazione delle modalità di valutazione.

Circa il primo aspetto, è raccomandata l'iscrizione nell' attivo patrimoniale del bilancio dei beni culturali per cui è possibile e non eccessivamente oneroso procedere alla loro valutazione. Viene pertanto consentito di eseguire la capitalizzazione asset-by-asset, anziché su un criterio all-or-nothing proprio del full capitalization approach proposto dall'IPSASB, la cui applicazione pratica incontra non pochi ostacoli.

In merito al secondo aspetto, lo standard FRS 30 permette alle organizzazioni di ricorrere a stime svolte internamente al fine della determinazione del 
valore di bilancio degli heritage asset ${ }^{11}$, senza la necessità di una verifica da parte di un auditor esterno accreditato. In particolare, le stime possono essere compiute da persone qualificate, quali curatori o esperti con conoscenze accertate.

Rispetto all'IPSASB e agli standard setter dei paesi anglosassoni, differente è l'impostazione seguita nella prassi contabile italiana. La proposta delle Commissione delle Aziende non Profit dell'allora Consiglio Nazionale dei Dottori Commercialisti (CNDC, 2004) raccomanda che i beni culturali siano valutati al costo storico. Tale scelta è dettata, oltre che dal fatto che nel nostro sistema il costo storico costituisce il criterio di base per le valutazioni di bilancio, dall'inopportunità di impiegare il fair value per asset che non sono destinati allo scambio e la cui iscrizione in bilancio deriva da misurazioni spesso soggettive ${ }^{12}$.

La preferenza per il costo storico viene disattesa qualora l'elemento patrimoniale sia stato acquisito a seguito di una donazione o di un lascito. In questo caso, esso dovrà essere stimato al "valore normale", ricorrendo (per quanto possibile) a riferimenti di mercato o procedendo mediante valutazioni effettuate da un perito indipendente.

Inoltre, si osserva che la Commissione stabilisce l'impossibilità di sottoporre ad ammortamento tutti i beni che hanno una vita utile indeterminata o estremamente lunga e non definibile (ad esempio, immobili di interesse storico-artistico e parchi naturali) o per i quali è impossibile quantificare la svalutazione (ad esempio, opere d'arte o altri beni di interesse culturale). Contestualmente, essa prescrive la creazione di una riserva vincolata di importo adeguato per far fronte ad eventuali oneri da sostenere per il mantenimento ed il ripristino dei beni, da utilizzare in caso di danneggiamenti o deprezzamenti ritenuti non ricorrenti.

Analizzando i criteri proposti nella prassi contabile, emergono non pochi limiti riguardanti la significatività dei risultati ottenuti attraverso il loro impiego o, addirittura, la concreta possibilità di applicazione.

Il costo storico costituisce un efficace parametro di riferimento nella valutazione dei beni culturali solo in situazioni estremamente circoscritte. In particolare, esso può essere rappresentativo del valore effettivo di un'opera d'arte, di una collezione o di un edificio storico in sede di prima rilevazione, ossia al momento dell'acquisto. Per operazioni di acquisizione lontane nel tempo, il costo storico spesso non è disponibile e comunque, anche nel caso lo sia, non appare un dato significativo, in quanto i beni culturali ed artistici spesso incrementano il proprio valore nel corso del tempo. Questa caratteristica esclude ogni possibilità di sottoporre tali beni ad un processo di ammortamento.

11. Circa i criteri di valutazione, il FRS 30 si limita a rinviare alle regole di carattere generale applicabili alle immobilizzazioni materiali, fornite nel FRS 15 "Tangible fixed assets".

12. Sulla valutazione al fair value dei beni culturali si veda Bagnoli e Manetti (2004). 
D'altra parte, essi presentano una vita utile molto estesa nel tempo che tende ad allungarsi ulteriormente qualora siano svolti periodici interventi di conservazione e restauro; ragion per cui gli heritage asset sono in genere classificati tra le immobilizzazioni a vita utile indefinita.

In aggiunta ai citati limiti segnaletici, il costo storico non è applicabile nelle circostanze, non infrequenti, in cui i beni culturali siano stati acquisiti dall'istituzione che li possiede a seguito di lasciti o di donazioni.

L'adozione del criterio del valore di mercato per la valutazione degli heritage asset, se da un lato permette teoricamente di superare talune carenze (soprattutto in termini di non rappresentatività del valore assegnato al bene) proprie del costo storico, dall'altro fa emergere diverse difficoltà sotto il profilo applicativo ${ }^{13}$.

In alternativa, la valutazione potrebbe essere effettuata ricorrendo al valore d'uso, procedendo cioè all'attualizzazione dei flussi di cassa derivanti dal bene. Ad esempio, si potrebbero utilizzare, come dati di input, le entrate monetarie relative ai biglietti di ingresso di un museo acquistati dai visitatori. Si osserva, tuttavia, che il criterio in discorso spesso non consente di determinare il valore di singoli oggetti d'arte in esposizione. In particolare, il valore d'uso non risulta applicabile per la valutazione di beni appartenenti ad ampie collezioni, in relazione ad ognuno dei quali non è possibile stimare i relativi flussi di cassa. Infatti, per la determinazione del valore di singoli oggetti che non siano stati acquisiti individualmente ma siano appartenenti ad una più vasta collezione, non appare impiegabile alcuno dei metodi richiamati, così come può dimostrarsi eccessivamente oneroso procedere ad una stima specifica.

In proposito, si rileva che nell'ambito degli studi di microeconomia sono stati sviluppati, a partire dagli anni ' 80 , alcuni modelli che, mediante il calcolo di flussi monetari, mirano a effettuare una valutazione dei beni culturali, intesi come beni pubblici con caratteristiche impure di non rivalità ed non escludibilità ${ }^{14}$.

Facciamo riferimento ai metodi di analisi econometrica delle preferenze espresse $^{15} \mathrm{e}$, in particolare, alla metodologia della contingent valuation.

13. Naturalmente, la valutazione a valori correnti implica la necessità di effettuare periodiche revisioni dei valori assegnati ai beni, le quali possono condurre a rivalutazioni o svalutazioni degli stessi. Le ipotesi in cui appare necessario dar luogo a queste ultime, date le caratteristiche evidenziate degli heritage asset, risultano circoscritte a situazioni di grave deterioramento fisico o di insorgenza di dubbi in merito all'autenticità dell'opera d'arte.

14. Le due caratteristiche di non rivalità e di non escludibilità attengono, rispettivamente, alla circostanza che il "consumo" del bene da parte di un attore non riduca le possibilità di consumo da parte degli altri e che nessun individuo possa essere escluso dalla fruizione del bene stesso.

15. Le metodologie per misurare il valore economico totale di un bene culturale possono, in termini generali, essere distinte in due categorie:

- le metodologie estimative basate sulle preferenze "rivelate" (osservate) da comportamenti degli utenti;

- le metodologie di stima basate sulle preferenze "espresse" dagli utenti. 
L'applicazione di quest'ultima presuppone l'effettuazione di un'indagine presso un campione selezionato di utenti, effettivi e potenziali, dalla quale è possibile ricavare (attraverso l'impiego di opportune tecniche di elaborazione statistica) una serie di valori monetari espressivi della "disponibilità a pagare" (willingness to pay) da parte degli intervistati per un determinato bene o complesso di beni culturali.

Senza entrare nel merito delle tecniche di contingent valuation, l'aspetto interessante al fine della nostra analisi è dato dal fatto che tale metodologia mira a giungere ad una valutazione di asset per cui non è agevole riscontrare un mercato attivo di riferimento e per i quali i criteri tradizionali proposti nella prassi contabile in molte circostanze non sono applicabili.

Tuttavia, si deve osservare che i modelli econometrici in questione presentano non pochi limiti ${ }^{16}$ che possono mettere in dubbio la correttezza e la significatività dei risultati ottenuti e, nello specifico, dei flussi monetari derivanti dalla willingness to pay.

A prescindere da questi limiti endogeni, si rileva che la metodologia in questione, se da un lato può costituire uno strumento per assumere decisioni strategiche a livello di singola organizzazione museale, dall'altro non può offrire un efficace supporto nella determinazione dei valori di bilancio dei beni culturali, date le inevitabili carenze informative dei soggetti (utenti effettivi e potenziali) presso i quali sono effettuate le indagini. Risultati più significativi possono sicuramente essere ottenuti con il coinvolgimento nella valutazione degli asset in oggetto di esperti del settore.

A tal proposito, si osserva che le variabili da prendere in esame così come le competenze richieste per effettuare la stima, possono essere molteplici e facenti capo a differenti aree scientifico-disciplinari.

Nel modello proposto da Forte-Mantovani (Forte e Mantovani, 2004, pp. 603-630) per la determinazione del valore dei beni delle arti figurative ${ }^{17}$, ven-

16. Le principali critiche alla metodologia della Contingent Valuation sono riconducibili al verificarsi dei seguenti fenomeni (Mazzanti 2003, pp. 98-99):

- distorsioni da comportamento strategico, se i rispondenti esprimono valutazioni che non riflettono i loro veri assetti di preferenza (con rischi di sottostime o sovrastime);

- distorsioni da scenario ipotetico o da mancanza di incentivi, se gli intervistati non hanno sufficienti incentivi a rispondere in modo veritiero rispetto alla proprie preferenze ed aspettative;

- distorsioni dovute al formato del questionario e al mezzo di pagamento scelto;

- distorsioni dovute a embedding effects, ossia a valutazioni che possono essere diverse se riferite ad un singolo bene preso separatamente oppure all'interno di un insieme più ampio (e.g. una collezione);

- distorsioni da informazione, legate principalmente alla quantità e alla qualità delle informazioni fornite agli intervistati prima di somministrare il questionario.

In proposito, si veda inoltre: Bedate, Herrero e Sanz (2009); Berrens et al. (2002); Candela e Scorcu (2004); Champ et al. (1997); Murphy et al. (2005); Throsby (2003); Venkatachalam (2004).

17. Circa la valutazione delle opere d'arte figurativa, si vedano, tra gli altri, Lazzaro e Mossetto (1997) e Gramp (1989). 
gono individuate una serie di variabili riguardanti i valori artistici (dell'autore, dell'opera e della tendenza), edonici (valori funzionali pratici, valori informativi extra-artistici, valori semiologici) e tecnici (dimensioni e materiali, provenienza e appartenenza o meno ad un contesto, rarità, firma, data e stato di conservazione). Accanto a queste variabili di natura endogena, non possono essere trascurate altre di carattere esogeno attinenti a fattori macro socio-economici (preferenze nazionali e fattori socio-politici, trend socio-culturali, cicli e trend macroeconomici) ed a fattori micro del mercato (caratteri liberisti o dirigisti del mercato, flussi informativi e marketing artistico).

Le variabili endogene, le quali attengono alla determinazione del "valore intrinseco" del bene (Mason, 2002), sovente differiscono o quantomeno assumono una diversa importanza relativa a seconda della tipologia del bene culturale considerato. A titolo esemplificativo, nel caso della stima di un reperto fossile conservato in un museo di storia naturale, assumono un significato preponderante nella determinazione dei valori artistici, elementi quali la completezza e la rarità del fossile, l'eccezionalità del giacimento fossilifero, l'accessibilità odierna della località di provenienza. Nella valutazione di un immobile di interesse artistico e storico (Forte e Mantovani, 2004, pp. 699-723), invece, acquistano un ruolo preminente variabili attinenti alle caratteristiche storicoartistiche e funzionali del bene e tra queste, qualora oggetto di valutazione sia una chiesa, non si può prescindere dalla considerazione del valore culturalesimbolico e spirituale-religioso del luogo di culto.

Le variabili esogene attengono a fattori di contesto, di natura sociale, politica ed economica, i quali - pur rivestendo un peso non trascurabile nel valore di bene culturale - non sempre sono adeguatamente considerati nelle perizie degli esperti (Mason, 2002). Senza entrare nel merito di questi fattori, ci limitiamo ad osservare che, laddove disponibile, un utile riferimento per la stima del valore di un bene culturale è costituito dai prezzi dei mercati d'asta (Candela e Scorcu, 1995, Scorcu e Zanola, 2011) e da quelli che si riscontrano sul mercato primario di galleristi, mercanti e collezionisti privati (Beltratti e Siniscalco, 1991).

Proprio per tenere compiutamente conto della pluralità di variabili da considerare nella valutazione di un bene culturale, è raccomandabile l'impiego di tecniche strutturate, quale ad esempio il c.d. Delphi method, nel quale è prevista la somministrazione di un questionario ad un gruppo di esperti (che può variare da un minimo di poche unità a più di una decina di individui) appartenenti a varie discipline (Ziglio, 1996; Carson et al., 1997; De Massimo, 1997). A tal proposito, si segnala che sono state recentemente formulate in letteratura alcune proposte volte a garantire una analisi sufficientemente ampia ed articolata, attraverso la partecipazione di molteplici competenze, di fonti informative differenziate ${ }^{18}$

18. Quagli e Ferrero (2010) richiamano, nella determinazione del fair value dei beni artistici da iscrivere in bilancio, una procedura di valutazione, ideata da una società composta da professionisti specializzati in ambiti artistici, giuridici, economici e tecnici, la quale si basa sulla media ponderata di tre diverse stime, ottenute da altrettante fonti di valutazione distinte: 1) i mercati d'a- 
Tab. 1 - Quadro di sintesi dei metodi applicabili per la valutazione dei beni culturali

\begin{tabular}{|c|c|c|c|}
\hline \multicolumn{2}{|c|}{ Metodi } & \multirow{2}{*}{$\begin{array}{l}\text { Punti di forza } \\
\text { - Maggiore oggettività } \\
\text { rispetto ad ogni altro } \\
\text { metodo }\end{array}$} & \multirow{2}{*}{$\begin{array}{l}\text { Punti di debolezza } \\
\text { - Non significativo per } \\
\text { acquisti remoti } \\
\text { - Non applicabile per } \\
\text { acquisti a seguito di } \\
\text { lasciti e donazioni }\end{array}$} \\
\hline $\begin{array}{l}\text { Metodologie } \\
\text { tradizionali } \\
\text { della prassi } \\
\text { contabile }\end{array}$ & Costo storico & & \\
\hline & $\begin{array}{l}\text { Valore corrente } \\
\text { di mercato }\end{array}$ & $\begin{array}{l}\text { - Valutazioni più } \\
\text { significative del costo } \\
\text { storico per acquisti } \\
\text { remoti e donazioni }\end{array}$ & $\begin{array}{l}\text { - Difficoltà applicative in } \\
\text { assenza di mercato attivo } \\
\text { di scambio o se con scarse } \\
\text { transazioni }\end{array}$ \\
\hline & Valore d'uso & $\begin{array}{l}\text { - Applicabile anche } \\
\text { nelle situazioni in cui } \\
\text { valore di mercato e } \\
\text { costo storico non sono } \\
\text { utilizzabili }\end{array}$ & $\begin{array}{l}\text { - Non applicabile in } \\
\text { assenza di entrate da } \\
\text { visitatori o se entrate } \\
\text { irrisorie } \\
\text { - Non determinabile il } \\
\text { valore di singoli beni in } \\
\text { ampie collezioni }\end{array}$ \\
\hline $\begin{array}{l}\text { Modelli di analisi } \\
\text { econometrica }\end{array}$ & $\begin{array}{l}\text { Contingent } \\
\text { valuation }\end{array}$ & $\begin{array}{l}\text { - Applicabile anche nelle } \\
\text { situazioni in cui "metodi } \\
\text { tradizionali" non sono } \\
\text { utilizzabili } \\
\text { - Mira a catturare il } \\
\text { "valore globale" dei } \\
\text { beni culturali }\end{array}$ & $\begin{array}{l}\text { - Flussi monetari c.d. } \\
\text { willingness to pay } \\
\text { altamente soggettivi e } \\
\text { spesso poco significativi } \\
\text { - Maggiore utilità per scopi } \\
\text { di decision making che } \\
\text { non di valutazioni di } \\
\text { bilancio }\end{array}$ \\
\hline $\begin{array}{l}\text { Valutazioni } \\
\text { di esperti }\end{array}$ & Metodo Delphi & $\begin{array}{l}\text { - Applicabile anche nelle } \\
\text { situazioni in cui "metodi } \\
\text { tradizionali" non sono } \\
\text { utilizzabili } \\
\text { - Si avvale del giudizio } \\
\text { di persone altamente } \\
\text { competenti }\end{array}$ & • Elevata soggettività \\
\hline
\end{tabular}

Fonte: nostra elaborazione.

e mediante l'applicazione di una vera e propria metodologia integrata ${ }^{19}$.

Senza entrare nel merito di queste proposte metodologiche, si rileva che esse possono costituire un'efficace (anche se onerosa) soluzione per la valutazione di beni culturali di notevole valore, nel caso in cui non siano impiegabili

sta e in particolare le stime effettuate dalle case d'asta; 2) la valutazione dell'opera eseguita da un perito esperto e indipendente; 3) il valore riscontrabile sul mercato degli oggetti d'arte.

19. Mason (2002) illustra una metodologia integrata, nella quale sono analizzati una pluralità di variabili socio-culturali (historical, cultural/simbolic, social, spiritual/religious, aesthetic) ed economiche [use (market) value, nonuse (non market) value, existence, option, bequest)] che concorrono a determinare il valore complessivo di un heritage asset, attraverso l'impiego di tecniche di valutazione sviluppate in ambiti disciplinari differenti. Si vedano, inoltre, i contributi di vari studiosi appartenenti a differenti aree disciplinari che affrontano la valutazione dei beni culturali secondo varie prospettive di indagine, raccolti in De La Torre M. (a cura di) (2002). 
i tradizionali criteri proposti dalla prassi contabile. Non può peraltro essere sottaciuto il fatto che il ricorso a tecniche che prevedono il coinvolgimento, nella stima, di una pluralità di esperti appartenenti a differenti ambiti disciplinari, non garantisce l'ottenimento di valori "oggettivi", rimanendo inevitabilmente elevata la componente soggettiva.

Una sintesi dei metodi applicabili nella valutazione dei beni culturali, con i relativi punti di forza e di debolezza è riportata nella Tab. 1 .

Il ragionamento sin qui condotto ha escluso, tranne specifiche situazioni, che il costo storico ed il valore d'uso possano rappresentare i criteri base per la valutazione del patrimonio museale. Dalla rassegna delle raccomandazioni della prassi contabile, emerge, al contrario, come il valore di mercato, laddove determinabile, rappresenti il punto di riferimento per le valutazioni del patrimonio museale, in particolare qualora i beni conservati siano a vita utile indefinita.

Nel prosieguo del presente lavoro presenteremo il caso del Museo di Storia Naturale dell'Università degli Studi di Firenze che si caratterizza per l'applicazione del metodo della stima degli esperti quale approssimazione del valore di mercato delle collezioni, così come indicato anche dallo standard britannico FRS 30.

Il caso in oggetto è stato scelto in quanto rappresenta il principale museo di storia naturale italiano per numero di esemplari. Esso, inoltre, ha intrapreso da alcuni anni un percorso di catalogazione ed inventariazione delle collezioni conservate al fine di indicare nel bilancio dell'Ateneo di Firenze il valore del proprio patrimonio.

\section{II Museo di Storia Naturale di Firenze: presentazione del case study}

Il Museo di Storia Naturale dell'Università degli Studi di Firenze (da qui in poi, il Museo), fondato nel 1775 dal Granduca Pietro Leopoldo (il nucleo dell'Orto Botanico risale tuttavia al 1545), è attualmente, con i suoi 8 milioni di esemplari, il maggiore museo naturalistico italiano per numero di esemplari ed uno dei più importanti a livello europeo (Barbagli e Pratesi, 2009).

Costituito da sei sezioni (Antropologia e Etnologia, Botanica, Geologia e Paleontologia, Mineralogia e Litologia, Orto botanico, Zoologia) dislocate nel capoluogo toscano, il Museo ospita reperti di grande valore scientifico e naturalistico.

Nel 1971 l'Università di Firenze stabilisce l'autonomia del Museo assegnandogli fondi, personale e spazi autonomi, nel 1984 le sezioni vengono ufficialmente riunite nel Museo di Storia Naturale dell'Università, mentre nel 2004 l'Ateneo avvia un profondo processo di trasformazione e potenziamento delle attività attraverso l'approvazione di un nuovo modello di governance e di un nuovo regolamento interno. 
Secondo l'art. 13 di tale regolamento, oggi il Museo gode di autonomia scientifica, amministrativa, finanziaria e contabile, tuttavia il soggetto giuridico è rappresentato dall'Università degli Studi di Firenze.

La mission è rintracciabile nel regolamento interno, secondo il quale il Museo "garantisce un servizio di ricerca scientifica e museologica, di promozione e divulgazione della cultura scientifica e naturalistica nonché di conservazione ed incremento delle collezioni".

Su indicazione degli organi di governo dell'Università di Firenze, negli ultimi anni è stato avviato un processo di catalogazione e successiva inventariazione delle collezioni, al fine rendere più trasparenti ed intelligibili le informazioni concernenti il patrimonio museale. La sua quantificazione, infatti, risulta particolarmente preziosa per stimare la posizione finanziaria e la solidità patrimoniale dell'Ateneo nel suo complesso e per fornire ai finanziatori pubblici (Stato, Regione ed Enti Locali) e privati (locale fondazione bancaria ed altre aziende), sia attuali sia futuri, elementi utili ai fini decisionali. A tale proposito, è opportuno evidenziare che la valorizzazione in bilancio ha riguardato sia i beni alienabili sia quelli inalienabili, pur nella consapevolezza che soltanto i primi potrebbero tramutarsi in future entrate monetarie qualora l'Ateneo optasse per la loro dismissione. Inoltre, grazie anche a questa scelta, come si evince dalla lettura del bilancio consuntivo annuale, l'Ateneo ritiene di assolvere in maniera più compiuta e trasparente ai propri doveri di accountability esterna ${ }^{20}$.

Inoltre, è necessario premettere che l'Ateneo, pur adottando tuttora una contabilità finanziaria di stampo pubblico, ha iniziato da alcuni anni un percorso di avvicinamento alla contabilità economico-patrimoniale. Tale scelta è legata sia all'assolvimento degli obblighi imposti dalla legge 240/2010 in tema di adozione della contabilità economico-patrimoniale e di quella analitico-gestionale all'interno degli Atenei italiani - già previste nei disegni di legge precedenti all'entrata in vigore della legge medesima - sia alla volontà di adottare tali approcci contabili quale fondamento di un nuovo modello di controllo direzionale. In questo senso, il processo valutativo del patrimonio è stato propedeutico all'introduzione del nuovo modello contabile e risulta particolarmente importante in un periodo di contrazione del finanziamento pubblico agli Atenei per attrarre nuove risorse da fonti pubbliche e private, destinate a finanziare le attività di conservazione, ampliamento e fruizione al pubblico delle collezioni.

20. Così come indicato dalla letteratura, la rappresentazione in bilancio del valore delle collezioni può contribuire, a determinate condizioni, all'assolvimento dei doveri di accountability interna ed esterna dei musei. In proposito si vedano: Sibilio Parri (2004b). Per un'analisi del management dei musei si vedano, tra gli altri, Chirieleison (2002); Jalla (2000); Matacena (2007); Sibilio Parri (2004a); Sibilio Parri (2007); Solima (2004). 


\subsection{La valutazione del patrimonio museale}

A partire dal 2005 il Museo ha promosso un'incisiva opera di completamento dell'inventariazione, unitamente a un processo di rivalutazione inventariale, consistente nell'attribuzione di un valore monetario ai singoli reperti o, forfettariamente, ad intere collezioni. Tale processo si è concluso nel 2009 con il risultato finale di una stima patrimoniale pari a 424 milioni di euro.

La valutazione delle collezioni è stata affidata ad esperti interni al Museo, in particolare ai curatori delle singole Sezioni o a team di esperti coordinati dai curatori.

La scarsa significatività del costo storico, connessa all'epoca di acquisizione dei reperti (variabile dal XVI al XXI secolo) e la contemporanea assenza, per larga parte di essi, di un prezzo di acquisto (si pensi, a titolo esemplificativo, ai reperti delle sezioni di zoologia e di botanica), hanno indotto i valutatori a propendere per il valore di mercato.

Il valore d'uso è stato scartato poiché si è in presenza di una scarsa significatività delle entrate da bigliettazione dato che il rapporto fra reperti esposti (fruibili al pubblico) e non esposti (fruibili soltanto agli studiosi) è attorno al $3 \%{ }^{21}$ e visto che il prezzo del biglietto è particolarmente contenuto. Inoltre, trattandosi di un Museo scientifico, la gestione è orientata anche alla conduzione di attività di ricerca che non prevedono un corrispettivo per l'accesso alle collezioni.

Tuttavia, anche nella determinazione del valore di mercato, a maggior ragione considerando la natura di museo scientifico, esistono difficoltà oggettive. Infatti, per gran parte dei reperti del Museo non esiste un mercato attivo con scambi regolari e continuativi. A tale proposito è opportuno notare che nel caso di un museo di storia naturale:

- il patrimonio museale è, da un punto di vista valutativo, eterogeneo e non omologabile, poiché i reperti conservati talvolta presentano le caratteristiche di beni pubblici, in altri casi di beni semi-pubblici ${ }^{22}$ e, in specifiche situazioni, di beni privati (Mazzanti, 2003, pp. 21-45);

- l'applicazione della contingent valuation presenta molteplici limiti connessi alla pressoché nulla conoscenza del reale valore degli oggetti da parte

21. Tale dato, peraltro, è in linea con i grandi musei internazionali, dove la percentuale di reperti esposti supera raramente il $5 \%$ e, in casi particolari, scende sotto all' $1 \%$. I valori riportati sono stati indicati dal Direttore del Museo di Storia Naturale di Firenze durante le interviste effettuate per l'analisi del caso. Per un confronto si veda anche: Tomea Gavazzoli (2003); Sabelli (2010).

22. I beni pubblici sono stati definiti in precedenza (si veda nota n. 14). Per quel che concerne i beni semi-pubblici si intendono quelli soggetti a congestione, ossia che hanno un consumo competitivo ma non escludibile, ossia con un'offerta limitata per cui un numero elevato di utenti genera rivalità. Si distinguono dai beni comuni che, al contrario, sono escludibili ma non rivali. Per approfondimenti: Forte (2007). 
della pubblica opinione che potrebbe condurre ad una stima distorta del patrimonio (Throsby, 2003, pp. 277-278);

- soltanto per alcune collezioni è ipotizzabile l'identificazione di un prezzo di mercato attraverso il ricorso a valori di scambio. Non è inusuale, infatti, la vendita di reperti fra musei scientifici in ambito nazionale ed internazionale. Inoltre, è possibile rintracciare valori di riferimento consultando, in casi specifici, case d'asta, riviste specializzate, cataloghi e gallerie;

- per i minerali e i fossili, ossia per i beni mineralogici e paleontologici, esistono dei floridi mercati internazionali (con periodiche borse) che rappresentano un valido riferimento per la valutazione degli esemplari;

- esistono massimali di polizze assicurative, relative o meno a prestiti di reperti, di alcune collezioni o parti di esse, stipulate negli ultimi anni. Non è infrequente che i massimali sottostimino il valore dei reperti coperti da polizza. In particolare, vi possono essere casi di sottostima quando l'onere assicurativo sia a carico del museo proprietario. Poche istituzioni, infatti, stanti le difficoltà finanziarie e l'andamento decrescente dei trasferimenti pubblici, sarebbero disposte a pagare alti premi per proteggersi da un danno potenziale sulle proprie collezioni difficilmente stimabile ed in presenza di reperti non sostituibili o riproducibili. Al contrario, raramente si inserisce un massimale di polizza più basso del reale valore del bene quando il pagamento dei premi sia a carico del richiedente. Ciò accade perché quest'ultimo vuole evitare che furti o danni al materiale preso in carico possano condurre a contenziosi legali con il proprietario dall'esito fortemente penalizzante;

- esistono alcune tipologie di beni (soprattutto quelle meno legate alla realtà scientifica) il cui valore di mercato è desumibile dalle indicazioni delle locali Soprintendenze (a loro volta ancorate a stime di esperti nazionali ed internazionali ed ai massimali delle polizze assicurative) che, come noto, operano quali organi periferici del Ministero per i Beni e le Attività Culturali;

- il Museo ha la possibilità di ricorrere a professionalità, interne (curatori) ed esterne (colleghi stranieri) in grado di assegnare alle collezioni e ai reperti il valore che si otterrebbe da una loro ipotetica alienazione ad altre istituzioni culturali, pubbliche amministrazioni o privati.

Sulla base di queste considerazioni il Museo ha realizzato una valutazione (in alcuni casi si è trattato di una più semplice rivalutazione) del patrimonio detenuto, ricorrendo ad esperti interni ed esterni, che ha riguardato gran parte delle collezioni. Dalla stima è stata esclusa la Sezione dell'Orto Botanico poiché legata alla conservazione di piante viventi per le quali l'attribuzione di un valore inventariale sarebbe stata priva di utilità e, forse, anche di significato.

In generale, la valutazione delle collezioni e dei reperti nel Museo di Storia Naturale di Firenze è avvenuta considerando i seguenti criteri di riferimento, perlopiù comuni a tutte le sezioni: 
- valore intrinseco (materia prima utilizzata e qualità della manifattura);

- antichità;

- rarità;

- stato di conservazione;

- collezione di appartenenza.

A tali criteri si sono aggiunti i seguenti parametri di riferimento:

- valore di mercato ove reperibile (prezzi di vendita nelle borse e nelle case d'asta nonché valutazioni attribuite nelle riviste specializzate, nelle gallerie e nelle esposizioni);

- esperienze di prestiti a mostre per le quali è stata richiesta polizza assicurativa ancorata al valore dei reperti;

- indicazioni e stime delle Soprintendenze o di altri enti specializzati di settore.

Inoltre, le valutazioni sono state mantenute nella fascia inferiore dell'intervallo dei possibili valori per ossequio ai principi della prudenza, pur in assenza di una reale possibilità di alienare - tranne alcune eccezioni - il patrimonio detenuto.

Una sintesi del processo valutativo, caratterizzato comunque dal minimo comune denominatore della stima degli esperti nelle cinque sezioni del museo - è riportata nella Tab. 2.

Tab. 2 - Quadro di sintesi delle valutazioni effettuate dalle singole sezioni del Museo

\begin{tabular}{|c|c|c|c|}
\hline & Valutatori $\quad$ Metodi utilizzati & $\begin{array}{l}\text { Criteri e parametri } \\
\text { di riferimento }\end{array}$ & $\begin{array}{l}\text { Stima complessiva } \\
\text { e numero reperti }\end{array}$ \\
\hline $\begin{array}{l}\text { Antropologia } \\
\text { e Etnologia }\end{array}$ & Curatori & $\begin{array}{l}\text { Criteri: valore intrinseco; antichità; } \\
\text { rarità; stato di conservazione; } \\
\text { collezione di appartenenza. } \\
\text { Parametri: stime della }\end{array}$ & $\begin{array}{l}30,8 \text { milioni di } € \\
\text { per circa } 44.000 \\
\text { reperti }\end{array}$ \\
\hline Botanica & Curatori & $\begin{array}{l}\text { Soprintendenza e di altri istituti } \\
\text { o archivi; massimali delle polizze } \\
\text { assicurative; pareri di esperti }\end{array}$ & $\begin{array}{l}132 \text { milioni di } € \\
\text { per circa } 4,6 \\
\text { milioni di reperti }\end{array}$ \\
\hline $\begin{array}{l}\text { Geologia e } \\
\text { Paleontologia }\end{array}$ & $\begin{array}{l}\text { Valore di mercato } \\
\text { desunto con stima } \\
\text { prudenziale di esperti }\end{array}$ & $\begin{array}{l}\text { stranıerı; offerte di acquisto } \\
\text { ricevute; costo pieno di acquisizione } \\
\text { del reperto; valori di borse e mostre } \\
\text { mercato; costo di riproduzione; } \\
\text { giudizio estetico. }\end{array}$ & $\begin{array}{l}16,3 \text { milioni di } € \\
\text { per circa } 220.000 \\
\text { reperti }\end{array}$ \\
\hline $\begin{array}{l}\text { Mineralogia e } \\
\text { Litologia }\end{array}$ & Curatori & & $\begin{array}{l}93,4 \text { milioni di } € \\
\text { per circa } 50.000 \\
\text { reperti }\end{array}$ \\
\hline Zoologia & Curatori & & $\begin{array}{l}152 \text { milioni di } € \\
\text { per circa } 3,6 \text { milioni } \\
\text { di reperti }\end{array}$ \\
\hline Orto botanico & I reperti (viventi) sono stati soltanto & talogati & \\
\hline
\end{tabular}

Fonte: nostra elaborazione. 


\section{Conclusioni}

Obiettivi del lavoro sono stati l'analisi dei principali metodi di valutazione dei beni culturali pubblici e, più in particolare, dei patrimoni museali pubblici secondo la dottrina e la prassi contabile e la presentazione di un caso di studio particolarmente significativo per la sua applicazione del metodo della stima degli esperti quale proxy del valore di mercato.

Rispetto al primo obiettivo, la disamina degli approcci proposti dalla prassi contabile ha fatto emergere la necessità di ricorrere a valori di mercato che, tuttavia, sono difficilmente desumibili. Le alternative proposte dai modelli econometrici e dalla valutazione degli esperti rappresentano strade percorribili per attribuire un valore di mercato ai beni culturali pubblici, anche se comportano un'elevata soggettività e complessità intrinseca del processo valutativo.

Rispetto al secondo obiettivo, si evidenzia che nel Museo di Storia Naturale di Firenze l'inventariazione è stata condotta per tutte le sezioni eccetto che per l'Orto botanico. In quest'ultimo caso i curatori si sono limitati alla catalogazione dei singoli pezzi, attraverso una descrizione di tipo qualitativo e l'assegnazione di un codice.

Per le restanti cinque sezioni si è attribuito un valore monetario alle collezioni alla luce della richiesta, proveniente dagli organi di governo dell'Università di Firenze, di contabilizzare il patrimonio museale all'interno dell'attivo patrimoniale dell'Ateneo.

Nel processo di misurazione, il criterio del valore d'uso è stato scartato per la scarsa significatività delle entrate da bigliettazione, nonostante i forti aumenti degli ultimi anni. Il costo storico, inteso come costo di produzione di campioni o reperti, è stato adottato raramente ed in situazioni circoscritte.

Con riferimento al valore di mercato è stato necessario fornire un'interpretazione particolare da parte del Museo. Infatti, in assenza di un mercato di riferimento per una larga maggioranza dei reperti, si è deciso di ricorrere al parere degli esperti interni e esterni per addivenire ad una stima attendibile. Gli esperti, a loro volta, hanno ancorato il processo valutativo ad alcuni criteri e parametri ricordati in precedenza e condivisi a livello internazionale dagli altri musei scientifici, anche riferendosi a valori che apparentemente non appartengono al novero di quelli di mercato (e.g. costo di restauro di determinati campioni). Anche nei casi nei quali il valore di mercato è chiaramente desumibile poiché esistono mercati di riferimento (e.g. nel caso dei fossili fuori dall'Italia), il valore è stato comunque corretto in ragione della limitatezza degli scambi su tale mercato o per tenere conto dell'unicità e della irripetibilità dei singoli pezzi.

La valutazione finale del patrimonio è dunque lontana dal rappresentare con certezza il patrimonio museale; essa tuttavia costituisce una ragionevole approssimazione della realtà, come del resto accade in tutti i processi valutativi, anche quando la valutazione abbia ad oggetto complessi aziendali funzionanti con esplicita finalità di lucro. 
Fra i possibili sviluppi del presente lavoro si segnala l'ampliamento del numero di case study per verificare l'applicabilità del metodo della stima degli esperti come proxy del valore di mercato. Tale verifica sarebbe necessariamente estesa alle valutazioni di esperti di diverse discipline (museologia, econometria, valutazione d'azienda) ricorrendo al metodo Delphi per giungere a valori attendibili e significativi.

Infine, un ulteriore possibile approfondimento è costituito dalla evidenziazione dei limiti del ricorso alle stime degli esperti per la valutazione del patrimonio museale, anche grazie al confronto con i risultati ottenuti da modelli econometrici - come quello della contingent valuation - e dalle metodologie proposte dagli economisti dell'arte (Candela e Benini, 1997; Candela e Scorcu, 2004).

\section{Riferimenti bibliografici}

Accounting standards board (ASB) (2009), FRS 30 Heritage Assets. (Londra: ASB).

Accounting standards board (ASB) (1999), FRS 15 Tangible Fixed Assets. (Londra: ASB).

Bagnoli L., Manetti G. (2004), Azienda-museo e non reciprocal transfer, in B. Sibilio Parri (a cura di), Misurare e comunicare i risultati. L'accountability del museo. (Milano: FrancoAngeli).

Barbagli F., Pratesi G. (a cura di) (2009), Museo di Storia Naturale dell'Università degli Studi di Firenze - Guida alla visita delle Sezioni. (Firenze: Edizioni Polistampa).

Barbati C., Cammelli M. e Sciullo G. (2006), Il diritto dei beni culturali. (Bologna: il Mulino).

Barton A. (2005), The conceptual arguments concerning accounting for public heritage assets: a note, Accounting, Auditing and Accountability Journal, 18, 3, pp. 434-440.

Bedate A.M., Herrero L.C. and Sanz J.A. (2009), Economic valuation of a contemporary art museum: correction of hypothetical bias using a certainty question, Journal of Cultural Economics, 33, 3, pp. 185-199.

Beltratti A. e Siniscalco D. (1991), Collezionisti, investitori e speculatori: la determinazione dei prezzi sul mercato dell'arte, Giornale degli economisti, 50, pp. 51-69.

Berrens R.P., Jenkins-Smith H., Bohara A.K., and Silva C.L. (2002), Further investigation of voluntary contribution contingent valuation: Fair share, time of contribution, and respondent uncertainty, Journal of Environmental Economics and Management, 44, 1, pp. 144-168.

Candela G., Benini M. (a cura di) (1997), Produzione e circolazione dell'informazione nel mercato dell'arte. (Bologna: Clueb).

Candela G., Scorcu A. (1995), Il prezzo dei dipinti: proposta per un numero indice delle aggiudicazioni d'asta. (Bologna, Clueb).

Candela G., Scorcu A. (2004), Economia delle arti. (Bologna: Zanichelli).

Caperchione E. (2001), Sistemi informativo-contabili nella pubblica amministrazione. (Milano: Egea).

Carman J., Carnegie G.D., and Wolnizer P.W. (1999), Is archaeological valuation an accounting matter?, Antiquity, 73, 279, pp. 143-148.

Carnegie G.D. and Wolnizer P.W. (1995), The financial value of cultural, heritage and scientific collections: an accounting fiction, Australian Accounting Rewiew, 7, 13, pp. $31-47$. 
Carnegie G.D. and Wolnizer P.W. (1999), Unravelling the rethoric about the financial reporting of public collections as assets. A response to Micallef and Peirson, Australian Accounting Rewiew, 9, 1, pp. 16-21.

Carson R.T., Mitchell R.C., Conway M.B. and Navrud S. (1997), Non Moroccan values for rehabilitating Fes Medina. (Washington DC: World Bank Report-Mimeo).

Champ P.A., Bishop R.C., Brown, T.C., and McCollum D.W. (1997), Using donation mechanisms to value nonuse benefits from public goods, Journal of Environmental Economics and Management, 33, 2, pp. 151-162.

Chirieleison C. (2002), La gestione strategica dei musei. (Milano: Giuffrè).

Christiansen J. (2004), Capital assets in governmental accounting reforms: comparing Flemish technical issues with international standards, European Accounting Review, 13, 4, pp. 743-770.

Cioppi E., Dominici S. (2009), How much are we willing to pay for a fossil?, Nature, 462, pp. 984-985.

Cioppi E., Tangocci F. (2008), Inventario delle collezioni paleontologiche del Museo di Storia Naturale di Firenze: obiettivi e modalità, Museologia Scientifica, Memorie,2, pp. 28-31.

CNDC-Commissione Aziende Non Profit (2004), Raccomandazione n. 6. Le immobilizzazioni. (Roma: CNDC).

De La Torre M. (a cura di) (2002), Assessing the Values of Cultural Heritage. (Los Angeles: The Getty Conservation Institute).

De Massimo G. (1997), Heritage conservation economics: a case study for Italy, in H. Coccossis and P. Nijkamp (editor), Planning for our cultural heritage. (London: Avebury).

Ferretti A. (2011), Diritto dei beni culturali e del paesaggio. (Napoli: Edizioni giuridiche Simone).

Forte F. (2007), Manuale di scienza delle finanze. (Milano: Giuffré).

Forte F. e Mantovani M. (2004), Manuale di Economia e Politica dei beni culturali, Vol. 1. (Catanzaro: Rubbettino).

Gramp (1989), Pricing the Priceless. Arts, Artists and the Economics. (New York: Basic Books).

Hone P. (1997), The financial value of cultural, heritage and scientific collections: a public management necessity, Australian Accounting Rewiew, 7, 13, pp. 38-43.

Hooper K., Kearins K. and Green R. (2005), Knowing the price of everything and the value of nothing. Accounting for heritage assets, Australian Accounting Rewiew, 18, 3, pp. 410-433.

IFAC/IPSASB (2006), Accounting for heritage assets under the accrual basis of accounting. Consultation paper. (New York: IFAC/IPSASB).

Institute of Chartered Accountants of New Zealand (ICANZ)-Financial Reporting Standards Board (FRSB) (2006), Comments on IPSASB consultation paper: Accounting for heritage assets under the accrual basis of accounting.

International Accounting Standard Board (IASB) (1989), Framework for the preparation and presentation of financial statements. (London: IASB).

Jalla D. (2000), Il museo contemporaneo. Introduzione al nuovo sistema museale italiano. (Torino: Utet).

Lacerra A. (2009), La contabilizzazione degli heritage assets, Rivista Italiana di Ragioneria e di Economia Aziendale (RIREA), 109, 5/6, pp. 360-373.

Lazzaro E. e Mossetto G. (1997), Le determinanti dei prezzi delle opere d'arte, in Candela G. e Benini M. (a cura di) (1997), Produzione e circolazione dell'informazione nel mercato dell'arte. (Bologna: Clueb). 
Lüder K. and Jones R. (2003), Reforming governmental accounting and budgeting in Europe. (PriceWaterhouseCoopers).

Mason R. (2002), Assessing Values in Conservation Planning: Methodological Issues and Choices, in De La Torre M. (a cura di), Assessing the Values of Cultural Heritage, The Getty Conservation Institute, pp. 5-30 (Los Angeles).

Matacena A. (2007), Mission, accountability e accreditamento nei musei: un percorso interpretativo. In B. Sibilio Parri (a cura di), Responsabilità e performance nei musei. (Milano: FrancoAngeli).

Mazzanti M. (2003), Metodi e strumenti di analisi per la valutazione economica del patrimonio culturale. (Milano: FrancoAngeli).

Micallef F. and Peirson G. (1997), Financial reporting of cultural, heritage, scientific and community collections, Australian Accounting Rewiew, 7, 13, pp. 31-37.

Ministero per i beni e le attività culturali (2001), D.M. 10 maggio 2001. Atto di indirizzo sui criteri tecnico- scientifici e sugli standard di funzionamento e sviluppo dei musei (Art. 150, comma 6, del D.Lgs. n. 112 del 1998), Roma.

Murphy J.J., Allen P.G., Stevens T.H. and Weatherhead D. (2005), A meta-analysis of hypothetical bias in stated preference valuation, Environmental and Resource Economics, 30, 3, pp. 313-325.

Onesti T. e Romano M. (2004), L'azienda-museo: informativa di bilancio e reporting del valore, in B. Sibilio Parri (a cura di), Misurare e comunicare i risultati. L'accountability del museo. (Milano: FrancoAngeli).

Organismo italiano di contabilità (OIC) (2005), OIC 16. Le immobilizzazioni materiali. (Roma: OIC).

Porter S. (2004), An examination of measurement methods for valuing heritage assets using a tourism perspective, Qualitative Research in Accounting \& Management, 1, 2, pp. 68-92.

Pozzoli M. (2005), Principi contabili internazionali ed enti locali. (Milano: FrancoAngeli).

Quagli A. e Ferrero F. (2010), Beni artistici: valutazione e rappresentazione in bilancio, Amministrazione \& Finanza, 5, pp. 6-9.

Romolini A. (2007), Il trattamento contabile dei beni artistici e culturali negli standard internazionali. In: R. Di Pietra (a cura di), Contabilità come bene pubblico. Interrelazioni economiche e politiche. Quaderno di Dottorato n. 15. Ciclo XXI. Siena.

Sabelli B. (2010), Museologia naturalistica. Trent'anni di «bicchieri a metà». (Bologna: Bononia University Press).

Scorcu A.E. e Zanola R. (2011), The "right" price for art collectibles: A quantile hedonic regression investigation of picasso paintings, Journal of Alternative Investments, 14 (2), pp. 89-99.

Sibilio Parri B. (a cura di) (2004a), Governare il museo. Differenti soluzioni istituzionali e gestionali. (Milano: FrancoAngeli).

Sibilio Parri B. (a cura di) (2004b), Misurare e comunicare i risultati: l'accountability del museo. (Milano: FrancoAngeli).

Sibilio Parri B. (a cura di) (2007), Responsabilità e performance nei musei. (Milano: FrancoAngeli).

Solima L. (2004), L'impresa culturale. Processi e strumenti di gestione. (Roma: Carocci).

Throsby D. (2003), Determining the value of cultural goods: how much (or how little) does contingent valuation tell us?, Journal of Cultural Economics, 27, 3-4, pp. 275285.

Tomea Gavazzoli M.L. (2003), Manuale di museologia. (Milano: Etas Libri). 
Valeri M. (2006), L'adozione di standard di funzionamento e sviluppo e la classificazione degli istituti museali, in B. Sibilio Parri (a cura di), Un modello di misurazione delle performance dei musei con particolare riferimento alla realtà calabrese. (Roma: Aracne).

Venkatachalam L. (2004), The Contingent Valuation Method: A Review, Environmental Impact Assessment Review, 24, 1, pp. 89-124.

Ziglio E. (1996), The Delphi method and its contribution to decision making, in M. Adler M. e E. Ziglio (editors), Gazing into the oracle: The Delphi method and its applications to social policy and public health. (London and Bristol (Pennsylvania): Jessica Kingsley). 